\title{
Gallium arsenide and indium arsenide surfaces produced by metalorganic vapor-phase epitaxy
}

\author{
L. Li, B.-K. Han, D. Law, M. Begarney, R.F. Hicks* \\ Department of Chemical Engineering, University of California, Los Angeles, CA 90095-1592, USA
}

\begin{abstract}
Thin films of GaAs and InAs were deposited on $\operatorname{GaAs}\left(\begin{array}{lll}0 & 0 & 1\end{array}\right)$ substrates by metalorganic vapor-phase epitaxy (MOVPE), and their surfaces were characterized by scanning tunneling microscopy (STM), X-ray photoelectron spectroscopy (XPS), and low-energy electron diffraction (LEED). Gallium arsenide surfaces produced in the MOVPE reactor at $570^{\circ} \mathrm{C}$ and a V/III ratio of 50 exhibit a $(1 \times 2)$ reconstruction, and are covered with weakly bound alkyl groups. Heating this material in flowing hydrogen in the reactor produces a variety of surface phases, depending on the sample temperature. These phases include $(2 \times 4)$ and $(4 \times 2)$ reconstructions, all of which are terminated with As or Ga dimers. The surfaces of InAs films grown by MOVPE exhibit these same phases. This study demonstrates that compound semiconductor surfaces formed in the MOVPE environment are nearly the same as those produced by molecular-beam epitaxy under ultrahigh vacuum conditions. (C) 1998 Published by Elsevier Science B.V. All rights reserved.
\end{abstract}

PACS: 81.15.Gh; 07.79.Cz; 68.35.Bs; 81.05.Ea

Keywords: Metalorganic vapor-phase epitaxy; Gallium arsenide; Indium arsenide; Scanning tunneling microscopy

\section{Introduction}

Many researchers have examined the reconstructions of III/V compound semiconductor $\left(\begin{array}{lll}0 & 0 & 1\end{array}\right)$ surfaces grown by molecular-beam epitaxy (MBE) in ultrahigh vacuum (see Ref. [1], and references therein). By contrast, comparatively little is known

\footnotetext{
* Corresponding author. Fax: + 1310206 4107; e-mail: rhicks@ucla.edu.
}

about the surface structures present under the technologically important conditions of metalorganic vapor-phase epitaxy (MOVPE) [2-8]. This is because MOVPE growth is carried out at 10-760 Torr, where only photon-based techniques for in situ surface analysis may be used. However, we have recently developed an apparatus that integrates MOVPE reactor with an ultrahigh vacuum surface analysis system [9-11]. This apparatus has allowed us to characterize the structure and composition of film surfaces as they evolve during growth. We find that the dimer-reconstructed 
surfaces are extremely stable under a hydrogen atmosphere. However, there are major differences between this environment and vacuum, most notably the presence of reactive alkyl species, that lead to interesting new surface phenomena. Below we describe some of our recent findings on the surface phases of MOVPE-grown GaAs and InAs(lo 001$)$ films.

\section{Experimental methods}

The GaAs( $\left(\begin{array}{lll}0 & 0 & 1\end{array}\right)$ wafers used in these experiments were miscut $0.5^{\circ}$ towards $\left[\begin{array}{lll}0 & 1 & 1\end{array}\right]$ and doped with $1 \times 10^{18} \mathrm{Si}$ atoms $/ \mathrm{cm}^{3}$ (AXT, Inc.). Gallium arsenide and indium arsenide films were deposited on these substrates using tri-isobutylgallium (TIBGa), tri-isopropylindium (TIPIn), tertiarybutylarsine (TBAs), in a hydrogen $\left(\mathrm{H}_{2}\right)$ carrier gas. The GaAs growth conditions were $570^{\circ} \mathrm{C}, 20$ Torr total reactor pressure, $4.5 \times 10^{-4}$ Torr TIBGa, a V/III ratio of 50 , and a space velocity of $30 \mathrm{~cm} / \mathrm{s}$ over the wafer surface (relative to $0^{\circ} \mathrm{C}$ and 760 Torr). The InAs growth conditions were the same, except that $5.0 \times 10^{-6}$ Torr TIPIn was substituted for the TIBGa. The InAs was deposited on $0.5 \mu \mathrm{m}$ of freshly grown GaAs, and the thickness was limited to less than $10 \AA$. Upon shutting the supply of organometallic precursors, terminating growth, the temperature was either lowered to $550^{\circ} \mathrm{C}$ for $20 \mathrm{~min}$ to produce the $(2 \times 4)$, or raised to $620^{\circ} \mathrm{C}$ for $20 \mathrm{~min}$ to produce the $(4 \times 2)$. Next the sample was cooled to $40^{\circ} \mathrm{C}$ at $1.3^{\circ} \mathrm{C} / \mathrm{s}$, the hydrogen flow stopped, and the reactor pumped down to $2 \times$ $10^{-7}$ Torr for transfer of the sample to the ultrahigh vacuum system.

The long-range order on the surface was determined with a Princeton Instruments low-energy electron diffractometer (LEED). The chemical composition of the surface was measured with a Physical Electronics X-ray photoelectron spectrometer (XPS), equipped with a multi-channel detector and a hemispherical analyzer. These data were acquired at a take-off angle of $35^{\circ}$ and a pass-energy of $23.5 \mathrm{eV}$. Scanning tunneling micrographs were obtained with a Park Scientific AutoProbe VP at $30^{\circ} \mathrm{C}$ with a sample bias of $-2.0 \mathrm{~V}$ and a tunneling current of $0.5 \mathrm{nA}$.

\section{Results and discussion}

\subsection{The $(2 \times 1) / c(4 \times 4)$ phase}

In the large-scale STM images of the gallium arsenide $\left(\begin{array}{lll}0 & 0 & 1\end{array}\right)$ surface taken immediately after MOVPE, one sees a regular array of terraces, indicative of step-flow growth [10]. The step edges run parallel to the $\left[\begin{array}{lll}0 & 1 & 0\end{array}\right]$ crystal axis and the average step width is approximately $300 \AA$. A close-up view of this surface is shown in Fig. 1a. Careful inspection of the image reveals that it is terminated with light gray rows, extending along the $\left[\begin{array}{lll}1 & 1 & 0\end{array}\right]$ direction. White lines are inserted in the figure to emphasize the row structure. The rows are $8 \AA$ apart, which corresponds to two unit cells of GaAs(0 0 1). However, no distinct periodicity is observed along the rows, therefore, the surface reconstruction is determined to be $(1 \times 2)$ by STM. LEED analysis of this surface produces a weak $\mathrm{c}(4 \times 4)$ pattern superimposed on intense $(1 \times 1)$ spots.

Next, the sample was annealed in vacuum at $350^{\circ} \mathrm{C}$ for $10 \mathrm{~min}$. This produced a sharp $\mathrm{c}(4 \times 4)$ LEED pattern. An STM image of this surface is shown in Fig. 1b. It contains two different structural domains, as indicated by the circles labeled $\mathrm{A}$ and $\mathrm{B}$. In circle $\mathrm{A}$, the light gray blocks are square, indicating that they are composed of a pair of arsenic dimers. In circle $\mathrm{B}$, the light gray blocks are rectangles, with the long axis parallel to the $\left[\begin{array}{lll}1 & 1 & 0\end{array}\right]$ direction. These blocks are formed by a group of three adjacent arsenic dimers. Inspection of the micrographs taken from four different GaAs wafers reveals that the two domains cover equal areas.

X-ray photoelectron spectroscopy of the GaAs surfaces reveals that only three elements are present after MOVPE growth: gallium, arsenic and carbon. Presented in Fig. 2 are XPS spectra of the C 1s peak as a function of annealing temperature in vacuum. The amount of surface carbon is below the detection limit of the spectrometer after annealing at $350^{\circ} \mathrm{C}$, indicating complete removal of carbon from the surface. On the other hand, the arsenic to gallium ratio does not change over this same temperature range, indicating that no arsenic desorbs below $350^{\circ} \mathrm{C}$. 

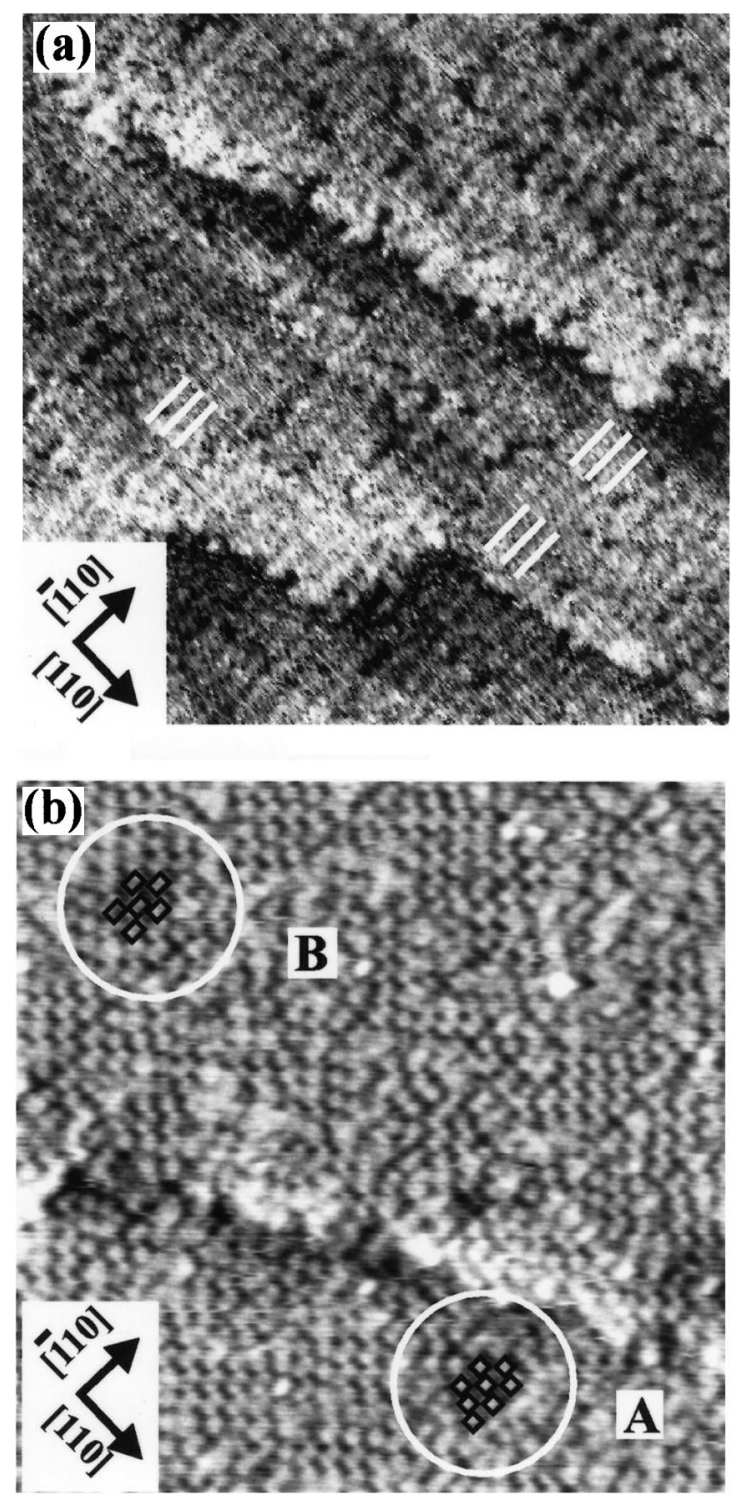

Fig. 1. (a) Scanning tunneling micrograph of the $\operatorname{GaAs}\left(\begin{array}{lll}0 & 0 & 1\end{array}\right)$ $(1 \times 2)$ surface immediately after transfer from the MOVPE reactor. Image size $=260 \times 260 \AA^{2}$. (b) Scanning tunneling micrograph of the $c(4 \times 4)$ reconstruction after annealing the GaAs crystal in vacuum at $350^{\circ} \mathrm{C}$. The image area is $320 \times 320 \AA^{2}$.

In order to identify the adsorbates present on the surface, infrared reflectance spectra have been collected before and after annealing the sample for $10 \mathrm{~min}$ at $100-400^{\circ} \mathrm{C}[10]$. Negative peaks at 2957 ,

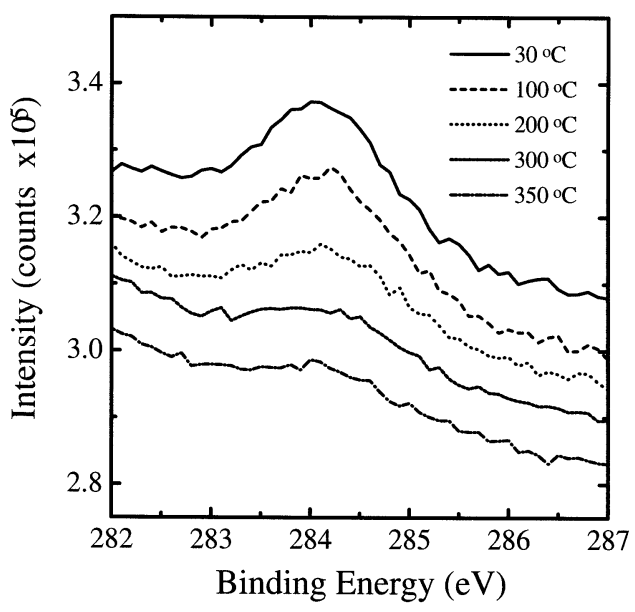

Fig. 2. X-ray photoemission spectra of the $\mathrm{C}$ 1s region after annealing a $\mathrm{GaAs}\left(\begin{array}{lll}0 & 0 & 1\end{array}\right)$ crystal in vacuum to temperatures between 100 and $350^{\circ} \mathrm{C}$.

2869 and $2926 \mathrm{~cm}^{-1}$ develop as alkyl groups desorb from the surface. These peaks are due to the asymmetric and symmetric stretching vibrations of $\mathrm{CH}_{x}$ speices [12-14]. No As and Ga hydrides are found, indicating that the surface is not terminated with hydrogen [15].

The results described above indicate that when the GaAs(l $\left.\begin{array}{lll}0 & 1\end{array}\right)$ film is removed from the MOVPE reactor it is terminated with a $\mathrm{c}(4 \times 4)$ structure that is covered with adsorbed alkyl species. The random distribution of these species on the surface produces the apparent $(1 \times 2)$ reconstruction observed by STM. In a previous paper, we proposed a model for the $(1 \times 2)$, which consists of rows of As dimers with chain lengths of 2 or 3 [10]. Interspersed between some of the dimer chains are single arsenic atoms that are terminated with a hydrocarbon radical, such as a methyl or ethyl group. These species tie up one of the dangling bonds of the As atom, so that it is three-fold coordinated, just like the other exposed atoms on the surface. Kamiya et al. [2-4] reported that under a flow of hydrogen and arsenic precursors, the GaAs surfaces exhibits a disordered $c(4 \times 4)$ structure. Similar results were found in GIXS studies by Kisker et al. [5-7]. The conditions used to produce these surfaces were analogous to those employed in this study. Therefore, we conclude that the disordered $c(4 \times 4)$ seen by these 
previous researcher is indeed the adsorbatecovered $(1 \times 2)$ structure identified herein.

\subsection{The $(2 \times 4)$ phase}

Shown in Fig. 3 is an STM image of the $(2 \times 4)$ reconstruction formed on the GaAs surface after growth and subsequent annealing in 20 Torr of flowing $\mathrm{H}_{2}$ at $550^{\circ} \mathrm{C}$. Light gray rows can be seen extending along the $\left[\begin{array}{lll}1 & 1 & 0\end{array}\right]$ direction, which are characteristic of the $(2 \times 4)$ structure [1]. However, it is evident that the surface does not exhibit a uniform appearance, due to a random distribution of white spots and black depressions within the gray rows. A black circle has been drawn around a pair of bright spots. Interestingly, these bright features disappear upon heating the sample above $300^{\circ} \mathrm{C}$ in vacuum. Analysis of the surface by XPS reveals that the concentration of carbon on the surface drops from 5.0 at $\%$ before heating to below 1.0 at $\%$ afterwards. This suggests that the white spots are due to adsorbed alkyl groups, as already discussed above for the $(1 \times 2)$ reconstruction. In addition, a white arrow has been inserted into Fig. 3 to highlight one

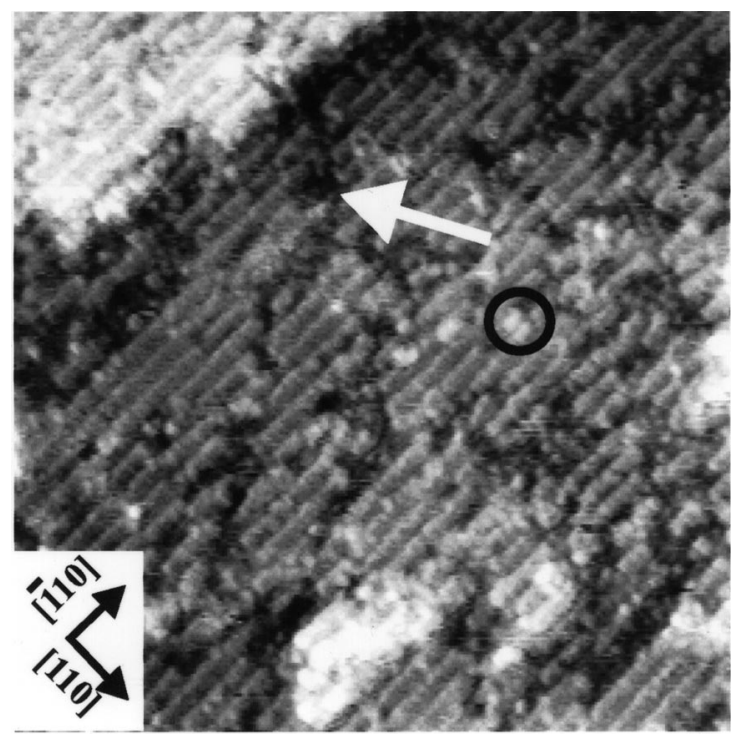

Fig. 3. Scanning tunneling micrographs of the $\operatorname{GaAs}\left(\begin{array}{lll}0 & 0 & 1\end{array}\right)$ $(2 \times 4)$ reconstruction prepared by annealing the GaAs crystal in the MOVPE reactor at 20 Torr $\mathrm{H}_{2}$ and $550^{\circ} \mathrm{C}$. Image size $=550 \times 550 \AA^{2}$. of the black depressions. Inspection of this spot reveals that a bunch of As dimers are missing from the rows. This is evidently due to desorption of arsenic during the heating step.

In contrast to what is observed in the MOVPE environment, we present in Fig. 4 an image of the $(2 \times 4) \mathrm{GaAs}(0001)$ surface after annealing in ultrahigh vacuum at $480^{\circ} \mathrm{C}$. This surface is uniform in appearance: no white spots or black depressions are observed. This sample was then returned to the MOVPE reactor and heated to $550^{\circ} \mathrm{C}$ for $20 \mathrm{~min}$ in 20 Torr of flowing hydrogen. After this treatment, XPS and STM analysis show that the $(2 \times 4)$ surface contains about 5.0 at $\%$ carbon as before, and has a structure identical to that presented in Fig. 3. Heating this sample above $300^{\circ} \mathrm{C}$ in vacuum reduces the carbon concentration below 1.0 at $\%$, and regenerates the image given in Fig. 4. This procedure can be repeated many times without any degradation of the surface quality. Thus, the $(2 \times 4)$ structure is quite stable in the hydrogen ambient of an MOVPE reactor.

The experiments described above for GaAs were repeated for InAs films. Similar results were

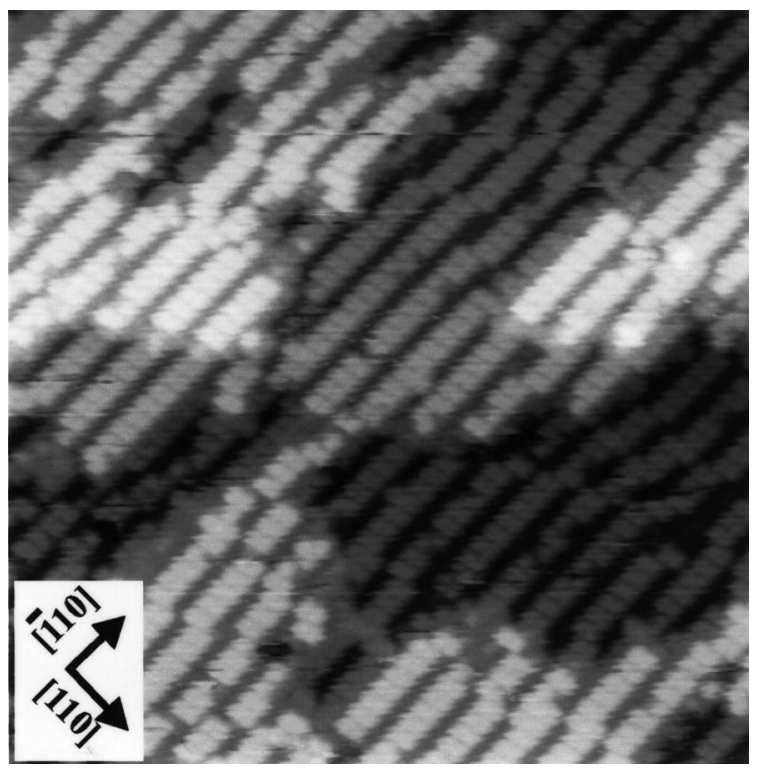

Fig. 4. Scanning tunneling micrographs of the $\operatorname{GaAs}\left(\begin{array}{lll}0 & 0 & 1\end{array}\right)$ $(2 \times 4)$ reconstruction prepared by annealing the GaAs crystal in vacuum at $480^{\circ} \mathrm{C}$. Image size $=285 \times 285 \AA^{2}$. 
obtained. After growth, the $\operatorname{InAs}\left(\begin{array}{lll}0 & 0 & 1\end{array}\right)$ surface is contaminated with alkyl species that are readily desorbed by heating in vacuum. Also, the sample can be transferred back and forth between vacuum and the MOVPE environment (20 Torr $\mathrm{H}_{2}$ and $550^{\circ} \mathrm{C}$ ) with no loss of the structural integrity of the $(2 \times 4)$ reconstruction.

\subsection{The $(4 \times 2)$ phase}

We have found that it is possible to prepare the metal-rich GaAs and InAs $(4 \times 2)$ reconstructions in the MOVPE reactor by annealing in 20 Torr $\mathrm{H}_{2}$ at $620^{\circ} \mathrm{C}$. Scanning tunneling micrographs reveal that these surfaces are analogous to the $(4 \times 2)$ reconstructions observed on films prepared by $\mathrm{MBE}$ $[1,16]$. Nevertheless, there are some notable differences. For example, patches of arsenic-rich $(2 \times 4)$ domains are found mixed in with the $(4 \times 2)$ phase. This is due to the background arsenic pressure in the MOVPE reactor when the susceptor and reactor walls are hot. The population of the $(2 \times 4)$ varies from sample to sample, and can be as high as $20 \%$ of the surface area. As an example, we show in Fig. 5 an STM image of the GaAs(l0 01$)\left(\begin{array}{ll}4 & 1\end{array}\right)$ surface. The $(4 \times 2)$ and $(2 \times 4)$ domains are labeled in the image. The $(4 \times 2)$ structure is characterized by rows running along the $\left[\begin{array}{lll}1 & 1 & 0\end{array}\right]$ direction, whereas the $(2 \times 4)$ domain contains rows running in the orthogonal direction [1]. Also note that the $(2 \times 4)$ is not uniform in appearance due to the presence of adsorbed alkyl radicals.

An STM image of the $(4 \times 2)$ reconstruction of InAs( $\left.\begin{array}{ll}0 & 0\end{array}\right)$, prepared in the MOVPE reactor, is shown in Fig. 6. Inspection of the STM image reveals that this structure consists of straight gray rows, extending in the $\left[\begin{array}{lll}1 & 1 & 0\end{array}\right]$ direction and spaced $16 \AA$ apart $(4 \times)$. In between these rows are dark stripes that are partially filled with light gray spots. These spots repeat every $8 \AA(\times 2)$. This structure is analogous to the InAs $(4 \times 2)$ prepared by MBE [16], except that in the latter case, the dark stripes are completely filled with the gray spots.

Arsenic-rich $(2 \times 4)$ domains are also evident in the STM images shown in Fig. 6. These arsenicrich unit cells aggregate together into small islands. Islands seen in the image vary in size over a wide

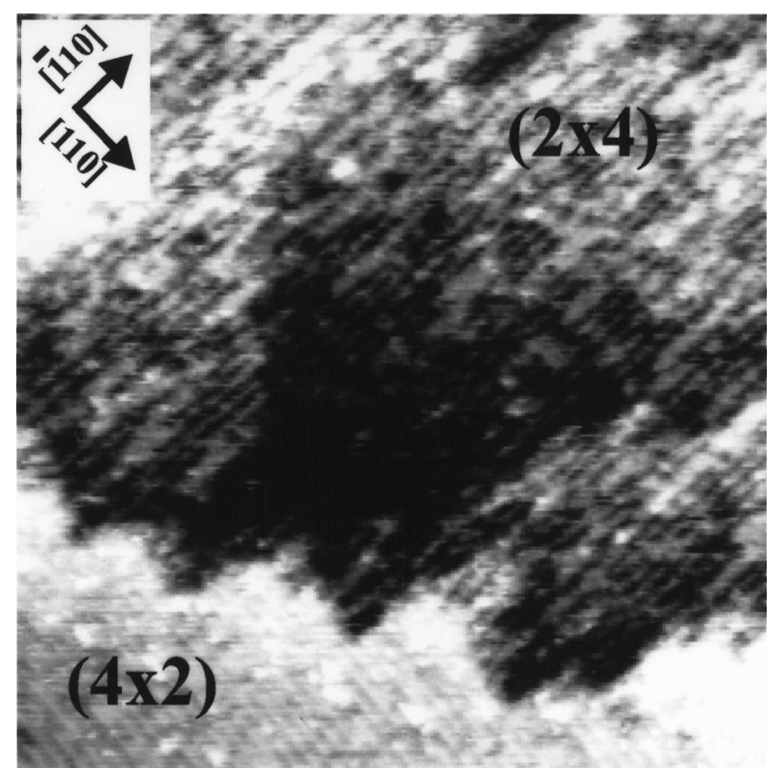

Fig. 5. Scanning tunneling micrograph of the $\operatorname{GaAs}\left(\begin{array}{lll}0 & 0 & 1\end{array}\right)$ $(4 \times 2)$ reconstruction after annealing the GaAs crystal in the MOVPE reactor at 20 Torr $\mathrm{H}_{2}$ and $620^{\circ} \mathrm{C}$. The image area is $650 \times 650 \AA^{2}$.

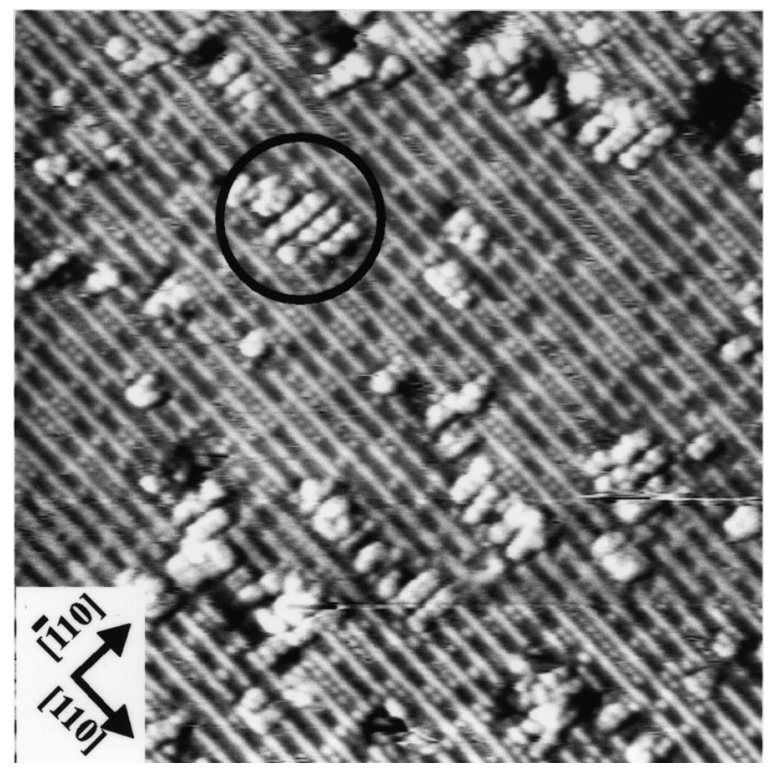

Fig. 6. ${ }^{\omega}$ Scanning tunneling micrograph of the $\operatorname{InAs}\left(\begin{array}{lll}0 & 0 & 1\end{array}\right)\left(\begin{array}{l}4 \\ 4\end{array}\right)$ reconstruction after annealing the GaAs crystal in the MOVPE reactor at 20 Torr $\mathrm{H}_{2}$ and $620^{\circ} \mathrm{C}$. The image area is $800 \times$ $800 \AA^{2}$. 
range. One of these domains is circled in the figure. The island contained within the circle extends about $32 \AA$ in the $\left[\begin{array}{lll}1 & 1 & 0\end{array}\right]$ direction and about $64 \AA$ in the $\left[\begin{array}{lll}1 & 1 & 0\end{array}\right]$ direction. These results are representative of the structures seen in many other images of

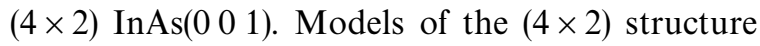
have been proposed in Ref. [17].

\section{Conclusions}

In previous studies of compound semiconductor surfaces in MOVPE reactors, using reflectance difference spectroscopy and X-ray scattering [2-8], it has been shown that a variety of structures can be produced, including $(1 \times 2), \mathrm{c}(4 \times 4),(2 \times 4)$ and $(4 \times 2)$ "like" phases. These structures are similar to, but not the same as, those observed in molecularbeam epitaxy. This study demonstrates that these differences arise from adsorbate-induced disorder, the presence of mixed domains, and defects within the unit cell.

\section{Acknowledgements}

Funding for this research was provided by the Office of Naval Research (N00014-95-1-0904), and by the National Science Foundation, Chemical and Thermal Systems (CTS-9531785) and Division of Materials Research (DMR-9804719).

\section{References}

[1] Q.K. Xue, T. Hashizume, T. Sakurai, Prog. Surf. Sci. 56 (1997) 1.

[2] I. Kamiya, H. Tanaka, D.E. Aspnes, L.T. Florez, E. Colas, J.P. Harbison, R. Bhat, Appl. Phys. Lett. 60 (1992) 1238.

[3] I. Kamiya, H. Tanaka, D.E. Aspnes, M. Koza, R. Bhat, Appl. Phys. Lett. 63 (1993) 3206.

[4] I. Kamiya, L. Mantese, D.E. Aspnes, D.W. Kisker, P.H. Fuoss, G.B. Stephenson, S. Brennan, J. Crystal Growth 163 (1996) 67.

[5] D.W. Kisker, G.B. Stephenson, P.H. Fuoss, F.J. Lamelas, S. Brennan, P. Imperatori, J. Crystal Growth 124 (1992) 1.

[6] F.J. Lamelas, P.H. Fuoss, P. Imperatori, D.W. Kisker, G.B. Stephenson, S. Brennan, Appl. Phys. Lett. 60 (1992) 2610.

[7] D.W. Kisker, G.B. Stephenson, I. Kamiya, P.H. Fuoss, D.E. Aspnes, L. Mantese, S. Brennan, Phys. Stat. Sol. (a) 152 (1995) 9.

[8] I. Kamiya, L. Mantese, D.E. Aspnes, D.W. Kisker, P.H. Fuoss, G.B. Stephenson, S. Brennan, J. Crystal Growth 163 (1996) 67.

[9] L. Li, B.-K. Han, R.F. Hicks, Appl. Phys. Lett. 73 (1998) 1239.

[10] B.-K. Han, L. Li, Q. Fu, R.F. Hicks, Appl. Phys. Lett. 72 (1998) 3347.

[11] L. Li, B.-K. Han, S. Gan, H. Qi, R.F. Hicks, Surf. Sci. 398 (1998) 386.

[12] L.J. Bellamy, The Infra-red Spectra of Comples Molecules, Wiley, New York, 1975, p. 13.

[13] P.E. Gee, H. Qi, R.F. Hicks, Surf. Sci. 330 (1995) 135.

[14] D.M. Joseph, R. Balagopal, R.F. Hicks, L.P. Sadwick, K.L. Wang, Appl. Phys. Lett. 53 (1988) 2203.

[15] H. Qi, P.E. Gee, R.F. Hicks, Phys. Rev. Lett. 72 (1994) 250.

[16] Q.K. Xue, Y. Hasegawa, T. Ogino, H. Kiyama, T. Sakurai, J. Vac. Sci. Technol. B 15 (1997) 1270.

[17] C. Kendrick, G. LeLay, A. Kahn, Phys. Rev. B 54 (1996) 17877. 